\title{
The effect of two weeks of spinal
} manipulative therapy and home stretching exercises on pain and disability in patients with persistent or recurrent neck pain; a randomized controlled trial

\author{
Anders Galaasen Bakken ${ }^{1 *}$, Andreas Eklund ${ }^{1}$, Anna Warnqvist ${ }^{2}$, Søren O’Neill ${ }^{3}$ and Iben Axén ${ }^{1}$
}

\begin{abstract}
Background: Recurrent or persistent neck pain affects a vast number of people globally, leading to reduced quality of life and high societal costs. Clinically, it is a difficult condition to manage, and treatment effect sizes are often moderate at best. Activity and manual therapy are first-line treatment options in current guidelines. We aimed to investigate the combination of home stretching exercises and spinal manipulative therapy in a multicentre randomized controlled clinical trial, carried out in multidiscipline ary primary care clinics.

Methods: The treatment modalities utilized were spinal manipulative therapy and home stretching exercises compared to home stretching exercises alone. Both groups received 4 treatments for 2 weeks. The primary outcome was pain, where the subjective pain experience was investigated by assessing pain intensity (NRS - 11) and the quality of pain (McGill Pain Questionnaire). Neck disability and health status were secondary outcomes, measured using the Neck Disability Indexthe EQ-5D, respectively. One hundred thirty-one adult subjects were randomized to one of the two treatment groups. All subjects had experienced persistent or recurrent neck pain the previous 6 months and were blinded to the other group intervention. The clinicians provided treatment for subjects in both group and could not be blinded. The researchers collecting data were blinded to treatment allocation, as was the statistician performing data analyses. An intention-to-treat analysis was used.
\end{abstract}

Results: Sixty-six subjects were randomized to the intervention group, and sixty-five to the control group. For NRS - 11, a B-coefficient of - 0,01 was seen, indication a 0,01 improvement for the intervention group in relation to the control group at each time point with a $p$-value of 0,305 . There were no statistically significant differences between groups for any of the outcome measures.

Conclusion: Based on the current findings, there is no additional treatment effect from adding spinal manipulative therapy to neck stretching exercises over 2 weeks for patients with persistent or recurrent neck pain.

Trial registration: The trial was registered 03/07/2018 at ClinicalTrials.gov, registration number: NCT03576846.

\footnotetext{
*Correspondence: Anders.Galaasen.Bakken@ki.se

1 Department of Environmental Medicine, Division of Intervention

and Implementation Research for Worker Health, Karolinska Institutet,

Nobels väg 13, S-, 17177 Stockholm, Sweden

Full list of author information is available at the end of the article
}

(c) The Author(s) 2021. Open Access This article is licensed under a Creative Commons Attribution 4.0 International License, which permits use, sharing, adaptation, distribution and reproduction in any medium or format, as long as you give appropriate credit to the original author(s) and the source, provide a link to the Creative Commons licence, and indicate if changes were made. The images or other third party material in this article are included in the article's Creative Commons licence, unless indicated otherwise in a credit line to the material. If material is not included in the article's Creative Commons licence and your intended use is not permitted by statutory regulation or exceeds the permitted use, you will need to obtain permission directly from the copyright holder. To view a copy of this licence, visit http://creativecommons.org/licenses/by/4.0/. The Creative Commons Public Domain Dedication waiver (http://creativeco mmons.org/publicdomain/zero/1.0/) applies to the data made available in this article, unless otherwise stated in a credit line to the data. 
Keywords: Manipulative therapy, Stretching exercises, Pain, Disability, RCT, NRS-11, EQ5D, Neck disability index, McGill pain questionnaire

\section{Background}

Musculoskeletal pain is responsible for the third-largest number of years lived with disability worldwide [1]. Its prevalence will likely rise due to an expected increase of such conditions in low-income and middle-income countries in the coming years [2] and a longer average life span worldwide [3].

Persistent or recurrent (also described as "chronic") pain is a prevalent condition, affecting $20 \%$ of the population globally $[4,5]$. Neck pain (NP) is a significant contributor to this group [6], as the condition is persistent or recurrent in $19-37 \%$ of the cases $[7,8]$. The consequences for NP sufferers are, among others, a higher risk of sick leave [9], a reduced ability to manage everyday life [10], and reduced mental and physical health-related quality of life [11].

NP is often labeled non-specific when no direct underlying cause is found, such as myelopathy or malignancy [12]. The pain is commonly thought to arise from painproducing structures such as the myofascia, cervical facet joints, or the disc [12]. The cause of persistent NP, on the other hand, is not all that clear. Persistent NP now falls under the category of chronic primary pain [4], defined in the ICD-11 as "pain ... that persists or recurs for longer than three months and is associated with significant emotional distress or significant functional disability (interference with activities of daily life and participation in social roles) and that cannot be better explained by another chronic pain condition" [4]. The consensus definition is that persistent or recurrent NP is located in the area of the neck, is constant or recurrent, with a minimum duration of 3 months [13-15].

There is little doubt that physical activity is beneficial for most persistent or recurrent pain patients $[16,17]$. It is also shown that some passive treatments reduce pain and have a place in the management of this patient group $[18,19]$. Recent guidelines for the management of persistent NP suggest multi-modal care such as stress selfmanagement, manipulation, mobilization, soft tissue therapy, high-dose massage, supervised group exercise, supervised yoga, supervised strengthening exercises, or home exercises [20-23].

Considering the worldwide suffering and cost of musculoskeletal pain, investigating and developing management approaches for this patient group is essential. As multi-modal treatment strategies are recommended for patients with persistent NP, investigating the commonly used treatment modalities and combinations of these could be useful contributions in managing this global epidemic.

In a clinical setting, patients will usually be offered a combination of treatments, advice, and exercise [24]. However, the specific combination of home stretching exercises and spinal manipulative therapy (SMT) has not been investigated for recurrent or persistent NP. We hypothesize that combining these two interventions will give a better outcome on pain and disability than stretching alone.

\section{Method}

\section{Setting and design}

Part of the study aimed to examine Heart Rate Variability (HRV) and Conditioned Pain Modulation, but this will be reported in separate publications. This study aims to determine the effects of a two-week treatment series consisting of i) home stretching exercises and SMT versus ii) home stretching exercises alone on pain and disability in a population of patients with persistent or recurrent NP. A sample size of 120 subjects was chosen based on a power calculation of the primary outcome of HRV [25], to be published seperately. We expected few drop-outs and therefore aimed for 130 .

The study was a multicenter randomized clinical trial, investigating adults with recurrent or persistent NP. Five multidisciplinary primary care rehab clinics in the Stockholm area recruited patients and contributed to the data collection. Recruitment began in October 2018 with the data collection ending in April 2020. The final follow-up questionnaires were answered in June 2020.

These clinics had chiropractors among their staff and were all part of the regional health service. This means that the patients paid part of the incurred fee (up to a maximum of $200 \mathrm{SEK} / 25$ USD per visit) to see any health care professional. The patient fee was capped at a total of 1150 SEK, after which all further costs were covered by the Swedish health care system. This also applied to the subjects in this study. The chiropractors providing care in this study were all licensed by the Swedish National Board of Health and Welfare.

\section{Recruitment}

Patients were recruited through one of several avenues:

i) Patients contacted the clinics directly and was informed about the ongoing study, ii) patients were referred from local general medical practices that had been informed about the ongoing study, iii) patients 
responded to project-specific online posts on the participating clinics' Facebook pages, iiii) patients responded to the monthly news letter from the clinic including information on the study and iiiii) patients responded to project-specific in-print advertising in local newspapers.

The possible subjects were screened for eligibility over the phone by the primary researcher using a protocol from Hallman et al. [26].

\section{Inclusion criteria}

- Presence of recurrent (at least one previous episode) and persistent (duration more than 6 months) NP [26]

- No chiropractic treatment the previous 3 months [27]

- Minimum 18 years of age

- Able to read and write Swedish.

\section{Exclusion criteria}

Conditions or medications that would affect the HRV measurements, such as

- cardiovascular disease

- hypertension

- diabetes

- pregnancy

- obesity $(\mathrm{BMI}>30)$

- steroids

- $\beta$-blockers

- antidepressants.

Also, subjects were excluded if they had

- serious, competing diagnoses, e.g. cancer, infection and serious trauma

- contra-indications to spinal manipulation, e.g. recent development of headache and/or dizziness, previous drop-attacks or acute cervical radiculopathy.

\section{Inclusion procedure}

Prior to inclusion, potential participants received verbal and written information concerning the study as per local ethics guidelines and were able to ask questions about the study to the principal researcher. Written, signed consent was obtained from all participants at this point.

Eligible subjects were booked in for all the study visits, 5 in total.

\section{Randomization}

The randomization sequence, using a 1:1 allocation ratio in randomly permuted blocks of different sizes, was generated off-site by a statistician, using SPSS version 27 [28]. Consecutively numbered sealed opaque envelopes were then prepared by a research assistant.

\section{First and subsequent visits}

A questionnaire was used to record demographic data and self-reported measurements (described below) at the baseline visit. The physical/biological baseline measurements were recorded by a research assistant before the patient saw his/her chiropractor. The chiropractor opened the allocation envelope and provided the allocated treatment modality.

All patients received four treatments in the study. In order to perform the follow-up measurements, participants were scheduled for five clinic visits and were measured again by the research assistant before their third and fifth treatment, respectively.

The procedures are extensively reported in the study protocol [25].

\section{Blinding}

Upon inclusion, all subjects were told that two different but common treatment modalities with similar clinical effects were tested in the study, but they were blind to what treatment the other group was receiving. Participants underwent the same examinations and number of treatments and had the same opportunities for support and to ask questions concerning their pain - the only systematic difference between groups was the allocated treatment.

The clinicians could not be blinded as they provided treatment for subjects in both groups.

The researchers collecting data were blinded to treatment allocation, as was the statistician performing data analyses.

\section{Procedures Interventions}

All subjects were scheduled for four treatments over the course of 2 weeks. The intervention dose and period were chosen as previous research had shown this would be sufficient to see a change in pain levels in a similar patient group [29, 30].

Both groups received a program of home stretch exercises with a documented effect on persistent neck pain [31], supplied in a leaflet with pictures and instructions (Attachment 1.). All subjects were asked to report the stretching in an exercise diary to measure adherence to the intervention. The diary is also found as part of Attachment 1. 
The intervention group received SMT in addition to home stretching exercises. SMT in this study was used as a term describing both mobilization and High Velocity Low Amplitude manipulation.

Mobilization and HVLA manipulation have similar effect sizes when used as a treatment for persistent NP in studies using pragmatic designs [32]. They are both favorable compared to other interventions [33], particularly in combination with multi-modal approaches [33, 34]. SMT combined with exercise has also been shown to improve the short term reduction of persistent NP, compared to exercise alone [18].

The treating chiropractor tailored treatment methods to suit each patient to adapt to factors like the subject's age, other diseases, and pain location. The treatment were given to any part of the spine as decided by the treating clinician. After completing the study, the chiropractor recorded treatment techniques used in each case from their patients' files.

\section{Outcome measures Pain (primary outcome)}

Pain intensity was quantified using an 11-point numeric rating scale (NRS-11), anchored by the descriptors 'No pain' (0) and 'Worst pain imaginable' [10], presented on paper and online questionnaires $[35,36]$. The qualitative characteristics of pain was assessed using the McGill Pain Questionnaire $[37,38]$ and the composite EQ-5D questionnaire was used to assess secondary effects of pain, such as emotions, previous pain experiences, and the effect on daily life [39-41]. The questionnaires have been shown to be reliable and valid in Swedish [35-41].

\section{Disability (secondary outcome)}

Using the Neck Disability Index (NDI) [42], the perceived level of disability was quantified by a summary score of the pain's effects on a patient's daily life. The instrument consists of 10 questions with a maximum score of 50 , where a higher score indicates higher degrees of perceived disability. The patient is asked to reflect on the degree of limitation in activities affected by their NP the previous week. The questionnaire is reliable and validated in Swedish [42].

\section{Health-related quality of life (secondary outcome)}

Health-related quality of life (HRQoL) reflects the overall persistent or recurrent pain experience. It was measured using EQ-5D, as it has been validated in patients living with persistent pain [43]. The EQ-5D defines the individual's health status by a single summary index ranging from 0 to 1 , where 0 corresponds to death, and 1 corresponds to full health $[41,44]$. The questionnaire is reliable and validated in Swedish [40, 41].

\section{Adverse reactions}

After the first visit, the subjects were asked to report any adverse reactions through a text message (SMS). They were asked whether they experienced a reaction to the first treatment, e.g. increased tenderness or fatigue in the neck, answered with an NRS-11 scale anchored by the descriptors No reaction' ( 0 ) and 'Worst reaction imaginable' [10] [45].

\section{Follow-up}

Digital outcome questionnaires were administered on the day of the third (1 week after the first treatment) and fifth treatment (2-4days after the fourth treatment), using a system managed by Survey \& Report by Artologic [46] through Karolinska Institutet.

Repeated measures of pain intensity (NRS-11) were recorded daily during the 2 week study period using SMS-Track ${ }^{\circledR}[45]$.

\section{Ethics}

This study investigated patients seeking care for their pain. A pure placebo trial was, therefore, not indicated [47]. As all subjects received home stretching exercises, a potentially beneficial intervention was provided. Four treatments were considered sufficient to detect a change, but not too burdensome if the patient did not benefit.

All study participants were assigned a unique number by a research assistant when included in the study. A code key connecting the participant's ID and the unique number was stored according to the Swedish National Board of Health and Welfare's requirements for the storage of journal documents.

All collected data will be stored for at least ten years, and individuals can only be identified with the code key. Only researchers directly affiliated with the project have access to the material.

The study was conducted in accordance with the Helsinki declaration [48]. Data was stored in accordance with European GDPR regulations.

\section{Statistical analysis}

Continuous variables were reported with means and standard deviations, categorical variables were reported as counts and percentages.

Intention to treat analysis was used.

Several subjects reported the McGill Questionnaire to be challenging to complete due to the numerous alternatives for quality of pain. Due to time constraints at the baseline visit, the subjects were instructed to skip the questions that did not relate to their pain instead of discussing them with the research assistant. Therefore, if a question was not answered, the subject did not experience the particular pain sensation. 


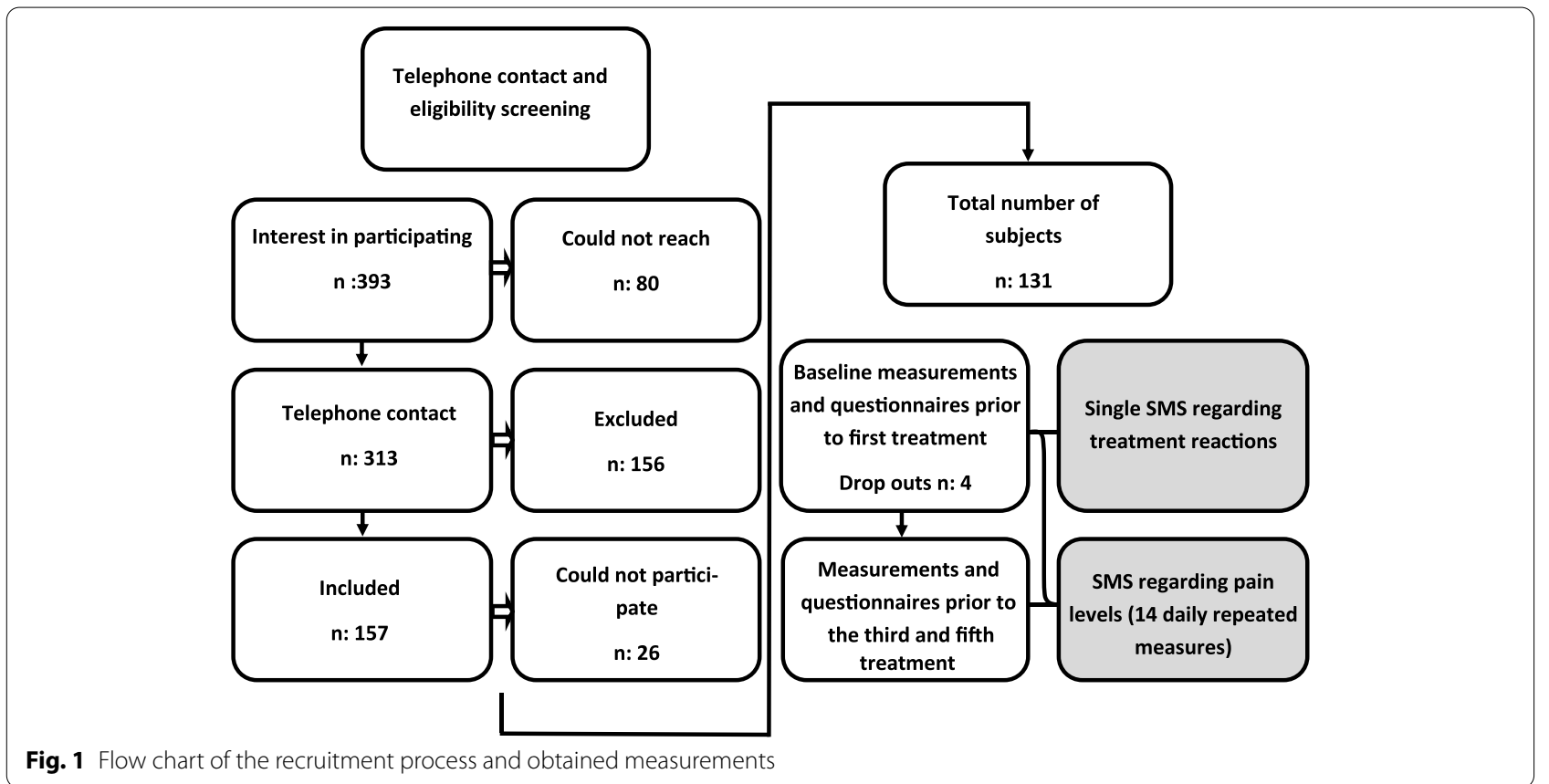

The NRS-11 data was incomplete with seven nonresponses, of which four concurrent (same date) pain scores could be obtained from the McGill questionnaire. For the final 3 missing observations, the Last Observation Carried Forward (LOCF) was used. A method of multiple imputations with fully conditional specification and twenty imputation rounds was used for NDI and EQ-5D to produce unbiased estimates with appropriate coverage [49].

Repeated measurements using all available time points were analyzed using a linear mixed effects model with person specific random intercept. A quadratic model was also estimated but the quadratic term did not improve model fit, judged by AIC (Akaike Information Criteria) and BIC (Bayesian Information Criteria). The parameter of interest was the interaction between group allocation and time, which can be interpreted as the difference in speed of change between the groups. Two additional analyses were performed; one adjusted for baseline values, the other also included age and gender.

The graphical representations were done using a quadratic model to allow for more flexibility and easier visual interpretation of the time effect interaction.

The change over the 2 weeks was calculated for all four outcomes; pain intensity, effective quality of pain, disability and quality of life. A minimal clinically important difference (MCID) was based on previous studies [50-53] and calculated as the percentage of subjects in each group reaching MCID. The values were as following; NRS-11: 2 points or more, NDI: 10 points or more, McGill: More than 5 points, EQ-5D: 0,03 points or more. The difference between groups in the probability of attaining MCID was estimated using logistic regression.

For linear and logistic regression analysis, both an unadjusted model, and models adjusted for baseline differences, as well as for age and gender, were estimated. The reported side effects are reported as proportions. A per-protocol analysis was performed as a sensitivity analysis, following the same method as the primary analysis for repeated measures.

$P$-values smaller than .05 were considered significant. The analysis was performed using SPSS 27 [28] and Stata version 15 (StataCorp. 2017).

\section{Results}

A total of 131 patients were included in the study. See attached flow chart (Fig. 1) for details.

Demographic and pain characteristics are presented in Table 1.

The two groups were similar at baseline, as reported in Table 1, except NRS-11, which differed with 0,51/10 points. An independent t-test showed this difference to be non-significant.

During the first week, four subjects dropped out, all in the control group. One dropped out due to a change in their work schedule following Covid-19, and two subjects were not pleased with receiving home stretching exercises only. One subject canceled for no reason. After the study period had ended, it was noted that two subjects 
Table 1 Demographics and pain characteristics of the study population at baseline, $N=131$

\begin{tabular}{|c|c|c|}
\hline & Intervention (66) & Control (65) \\
\hline Age, Mean (sd) & $57(14,0)$ & $57,5(13,7)$ \\
\hline Female, $n(\%)$ & $37(56)$ & $36(55)$ \\
\hline \multicolumn{3}{|l|}{ Pain duration } \\
\hline 1. Less than 6 months, $n$ (\%) & $0(0)$ & $1(2)$ \\
\hline 2. 6-12months, $n(\%)$ & $8(12)$ & $10(16)$ \\
\hline 3. Several years, $n(\%)$ & $57(88)$ & $51(82)$ \\
\hline \multicolumn{3}{|l|}{ STarT Back categories } \\
\hline 1. Low risk, n (\%) & $47(80)$ & $48(79)$ \\
\hline 2. Medium risk, $n$ (\%) & $7(12)$ & $11(18)$ \\
\hline 3 High risk, $n(\%)$ & $5(9)$ & $2(3)$ \\
\hline \multicolumn{3}{|l|}{ If seeing a chiropractor before, how effective was it? } \\
\hline 1. Never seen a chiropractor before, $n(\%)$ & $12(19)$ & $11(17)$ \\
\hline 2. Good or excellent, $n(\%)$ & $39(60)$ & $35(55)$ \\
\hline 3. No difference, $n(\%)$ & $14(22)$ & $17(27)$ \\
\hline 4. Got worse, n (\%) & $0(0)$ & $1(2)$ \\
\hline \multicolumn{3}{|l|}{ Type of occupation } \\
\hline 1. No job, n (\%) & $19(29)$ & $20(31)$ \\
\hline 2. Mostly hard labor, varied or standing, $n(\%)$ & $17(27)$ & $15(23)$ \\
\hline 3. Mostly sitting, $n(\%)$ & $30(46)$ & $30(46)$ \\
\hline \multicolumn{3}{|l|}{ Similar pain previously } \\
\hline 1. No, n (\%) & $35(53)$ & $34(56)$ \\
\hline 2. Yes, n (\%) & $31(47)$ & $27(44)$ \\
\hline Arm pain, $n(\%)$ & $42(65)$ & $36(57)$ \\
\hline Pain in the midback, $n$ (\%) & $39(61)$ & $37(62)$ \\
\hline Pain in the low back, $n(\%)$ & $39(62)$ & $37(59)$ \\
\hline \multicolumn{3}{|l|}{ Sick leave the previous year } \\
\hline Do not work, n (\%) & $13(20)$ & $18(28)$ \\
\hline No, $n(\%)$ & $47(71)$ & $41(63)$ \\
\hline Yes, between 1 and 7 days, $n$ (\%) & $3(5)$ & $2(3)$ \\
\hline Yes, between 8 and 14 days, n) (\%) & $3(5)$ & $0(0)$ \\
\hline Yes, more than 15 days, $n(\%)$ & $0(0)$ & $4(6)$ \\
\hline Expect to improve (0-10), Mean (std) & $6,0(2,2)$ & $5,8(2,3)$ \\
\hline
\end{tabular}

had received treatment though they were part of the control group.

See Table 2 and supplementary files 1, 2, 3 and 4 for an overview of outcome measures at baseline and at two-week follow-up. Both groups showed improvements in pain intensity (NRS-11), affective quality of pain (McGill questionnaire), disability (NDI) and quality of life (EQ-5D).

There were no statistically significant differences between the two groups in change scores for any of the outcome measures.

Additional models were tested, first using baseline differences as a covariate, secondly age and gender. Finally, a per protocol analysis was conducted, neither changed the overall estimates or precision and are therefore not reported here.

In the Supplementary files 1, 2, 3 and 4, daily development of mean pain intensity (NRS-11), McGill scores, Neck Disability Index and EQ5D scores with Confidence Intervals for both groups from baseline to the end of the intervention period ( 2 weeks) are presented graphically. The graphs are produced using a quadratic model.

Besides the crude difference between follow-up and baseline, we also calculated the proportion of subjects that reached MCID for each outcome. As reported in Table 3, some subjects in both groups reached a MCID for all measurements ranging from 18 to $38 \%$. There are no statistically significant differences between the two 
Table 2 Mean effect and B-coefficient for all outcome measures

\begin{tabular}{|c|c|c|c|c|c|c|}
\hline & Intervention & Control & B & $\mathrm{Cl}$ & & $P$-value \\
\hline \multicolumn{7}{|l|}{ NRS-11 } \\
\hline Baseline, mean (n) & $4,7(65)$ & $4,2(64)$ & & & & \\
\hline After 2 weeks (128) (n) & $3,6(66)$ & $3,1(62)$ & & & & \\
\hline Difference BL-2 weeks & $-1,1$ & $-1,1$ & $-0,0$ & $-0,0$ & 0,0 & 0,31 \\
\hline \multicolumn{7}{|l|}{ McGill questionnaire } \\
\hline Baseline (n) & $23,7(64)$ & $23,2(64)$ & & & & \\
\hline After 2 weeks $(n)$ & $22,8(65)$ & $21,3(61)$ & & & & \\
\hline Difference BL-2 weeks & $-0,9$ & $-1,9$ & 0,5 & $-0,6$ & 1,6 & 0,38 \\
\hline \multicolumn{7}{|l|}{ EQ5D } \\
\hline Baseline (n) & $0,897(66)$ & $0,903(65)$ & & & & \\
\hline After 2 weeks (n) & $0,897(62)$ & $0,899(57)$ & & & & \\
\hline Difference BL-2 weeks & $-0,007$ & 0,006 & 0,001 & $-0,012$ & 0,013 & 0,92 \\
\hline \multicolumn{7}{|l|}{ Neck Disability Index } \\
\hline Baseline (n) & $22,6(57)$ & $21,7(58)$ & & & & \\
\hline After 2 weeks (n) & $20,5(58)$ & $19,8(55)$ & & & & \\
\hline Difference BL -2 weeks & $-2,1$ & $-1,9$ & $-0,0$ & $-0,3$ & 0,1 & 0,45 \\
\hline
\end{tabular}

groups for any measurement. The adjusted analysis, including baseline, age and gender, is not included in the table as it did not change the overall effect and statistical significance.

Further, a per-protocol-analysis did not significantly affect results (results not shown).

The adherence to the stretching protocol was good, as shown in Table 4. Training diaries from $90 \%$ of the entire study population were obtained. Of these, $77,8 \%$ in the intervention group and $76,4 \%$ in the control group adhered to the stretching regimen at least 13/14days. All participants reported that they did their stretching exercises at least 10 out of 14 days.

\section{Adverse events}

Four intense adverse events were reported in the study, defined by $\geq 8$ (NRS-11) [54], three in the intervention group, and one in the control group. More adverse incidents were reported in the intervention group, with a

Table 3 Proportion of individuals reaching minimal clinically important difference for the different outcome measures and the difference between groups

\begin{tabular}{lllll}
\hline & Intervention & Control & OR & $P$-value \\
\hline $\begin{array}{l}\text { NRS-11 } \\
\quad \text { Reached MCID, n (\%) }\end{array}$ & $24 / 64(37)$ & $22 / 59(38)$ & 1,0 & 0,98 \\
McGill \\
$\quad$ Reached MCID, n (\%) & $15 / 62(24)$ & $11 / 57(18)$ & 1,4 & 0,46 \\
EQ5D \\
$\quad$ Reached MCID, n (\%) & $14 / 66(21)$ & $19 / 65(29)$ & 0,6 & 0,30 \\
$\quad$ NDI \\
$\quad$ Reached MCID, n (\%) & $15 / 66(22)$ & $18 / 65(28)$ & 0,7 & 0,45 \\
\hline
\end{tabular}

mean pain intensity (NRS-11) of 2,75 compared to 1,22 in the control group. There were no statistically significant differences between the two groups.

\section{Discussion}

The current study was undertaken to investigate whether four sessions of SMT combined with 2 weeks of stretching exercises was superior to stretching exercises alone, in a group of chronic NP patients. Both groups improved with treatment, but we found no statistical differences for any of our outcome measures between the two groups.

Previous studies have found a combination of home exercises and SMT superior to home exercises alone $[19,34]$, and most current guidelines recommend multimodal care, including SMT and exercises [20-23]. However, the combined effect of SMT and home stretching exercises specifically has not been investigated in detail in chronic NP.

The stretching exercises used in this study were developed by Ylinen et al. [31] in a study comparing home stretching exercises with manual therapy. They found that both interventions considerably decreased neck pain

Table 4 Adherence to stretching exercises as reported in exercise diaries

\begin{tabular}{llllll}
\hline $\begin{array}{l}\text { Days of doing } \\
\text { stretching (out of } \\
\text { 14) }\end{array}$ & $\mathbf{1 0}$ & $\mathbf{1 1}$ & $\mathbf{1 2}$ & $\mathbf{1 3}$ & $\mathbf{1 4}$ \\
\hline $\begin{array}{l}\text { Group } \\
\text { Intervention, } \mathrm{n}(\%)\end{array}$ & $4(6,3)$ & $0(0,0)$ & $10(15,9)$ & $10(15,9)$ & $39(61,9)$ \\
$\quad$ Control, $\mathrm{n}(\%)$ & $0(0,0)$ & $7(12,7)$ & $6(10,9)$ & $6(10,9)$ & $36(65,5)$ \\
\hline
\end{tabular}


and disability in women with persistent or recurrent neck pain. The difference in effectiveness between the interventions was minor. We hypothesized that SMT and home stretching exercises combined would have a better effect than home stretching exercises alone after a twoweek treatment period. However, the results rather imply that providing stretching only is as effective as combining it with SMT, and that perhaps the patient's own preference should guide the treatment choice.

A population with persistent or recurrent pain will typically have periodic changes in pain intensity [55], and the fluctuations in pain and disability in this study could be due to normal variations in pain. Overall, an improvement during the study for all measurements was seen in both groups when adjusted for baseline differences in pain intensity.

The number of people reaching MCID did not differ statistically between groups. Just below $40 \%$ reached this threshold in pain intensity (NRS-11), but only around $20 \%$ for the impact of pain quality (McGill questionnaire). This is an interesting observation as they are both measuring the subject's pain experience. It appears that pain intensity is more readily affected by the intervention than the sensory and affective pain experience, measured using the McGill Questionnaire. However, an absolute consensus on MCID values does not exist, so care should be taken when comparing these values. It was not expected that a large part of the study population would reach MCID, considering the chronicity of their pain and the limitations of a two-week study period.

It was observed that the SMT and stretching group experienced more adverse reactions than the group receiving stretching only. The difference was not statistically significant, but is not surprising considering previous research. It is estimated that about $50 \%$ of patients experience minor to moderate adverse events after any manual treatment, particularlyafter the first visit [54]. Since the risk of major adverse events is low, and that these adverse events are known to be short-lasting [56], adverse events were only monitored after the first study visit. Adverse events in this study were few and mainly mild, in line with previous studies $[54,56]$ confirming that both interventions are safe.

Adherence to the home stretching exercises was good in both groups (all subjects reporting compliance at least 10 out of 14 days, and $77 \% 13$ or 14 days). In the original stretching study, the investigator asked the subjects to perform the stretching exercises five times a week [31]. We chose to recommend the exercises to be performed daily as the intervention period was only 2 weeks. We also accounted for some forgetfulness among the subjects based on clinical experience. Previous research has shown that getting people to perform their rehab exercises is one of the most difficult challenges in clinical health interventions $[57,58]$. We propose that adherence to the program was vital in the observed improvement. Filling in a diary probably improved adherence to the protocol.

For pain intensity (NRS-11), there was a difference between the treatment groups at baseline. This happened by chance in the randomization process and did not significantly affect the estimates when adjusted for.

\section{Methodological considerations Weaknesses}

It was impossible to blind the therapists performing the treatments. This could possibly lead to bias favouring the intervention group. However, the therapists received written and verbal instructions on how to interact in a similar fashion with both groups to minimize this risk. As there was no significant advantage of the intervention group, this potential bias was considered to be low.

Most of the participants had already been to the clinic at a previous time point. A possible selection bias may have occurred as many of the subjects already had a good experience with a previous chiropractic treatment. This is an unknown effect, not directly related to a specific treatment or clinician. If the subject had seen a therapist for their neck pain previously and experienced a good effect, this could have had a substantial impact on the expectations of a treatment effect. However, it can be argued that if a therapist was already managing a patient with neck pain successfully, i.e. they have a good relationship, and the patient is experiencing that the treatment regime helps them manage their life with pain, then participation in this study would not be needed. The improvement in pain and disability in the intervention and control groups was similar in this study. The first and obvious conclusion, is that the two treatments have equal clinical benefit to patients, which is not in line with previous publications [19, 34]. The current findings, however, could be affected by factors such as a flooring effect, the relatively short treatment period for such a chronic patient cohort, or the chronicity of this patient group.

We had no minimum inclusion value for baseline measurements, and generally speaking, the study population had low levels of pain and disability. This was not done to include all aspects of persistent or recurrent NP patients, as it is a diverse group. For NRS-11 i.e., a level of 1 or 2 out of 10 at baseline will have limited the improvement to 1 or 2 points, respectively. This could possibly have limited the difference between groups.

The inclusion criteria explicitly stated recurrent or persistent NP, where pain fluctuations are the norm [55]. As 
the recruiting was done up to 5 weeks before the intervention period, patients may have been in more pain when recruited but experienced a normal fluctuation for the better before the study started. Thus, changes seen in his study are probably not due to regression to mean, but this cannot be ruled out.

Four treatments were chosen as it has been seen from a previous study [29] that a definite improvement observed at the fourth treatment usually is related to an improvement after 3 months. However, that study population consisted of patients with low back pain, and among these, only a group had persistent or recurrent pain. Also, the subjects sought chiropractic treatment, with no limitations regarding treatment intervention. It is possible that persistent or recurrent NP is more resistant to SMT, and that our study population had lower expectations to the intervention as they did not actively seek care at a chiropractic clinic.

These weaknesses may explain why the observed effect in the study population differs from previous studies, exemplified by the study by Maiers et al. [34] that had a minimum NRS-11 of 3 as inclusion criteria on NRS-11 and tested an intervention lasting twelve weeks.

\section{Strengths}

Blinding seems to have been successful, and the research assistant and statistician were blinded to the group allocation. The study population was blinded to the other group intervention.

Both groups received the same amount of time and attention from the chiropractors. The control group underwent a standard palpation examination as if the chiropractor intended to give manual treatment. Advise and reassurance were given in both groups.

Measuring pain levels and the affective quality of pain is essential for capturing the chronic pain experience. Using multiple questionnaires, such as NRS-11, McGill questionnaire, and EQ-5D together covers the main aspects of chronic pain.

The response rate was excellent, with a small number of dropouts and an exercise diary to investigate compliance. This showed a strong dedication to the stretching regime.

\section{External validity}

This was a pragmatic study, applicable for all clinicians working with persistent or recurrent NP patients. The study was perfomerd in a normal clinical setting, reflected in the heterogenic demographics among the study population. It mirrored standard treatment strategies to allow for conclusions on effect.

\section{Conclusion}

Daily stretching exercises with and without added spinal manipulation were associated with some clinical improvement over a two-week period, but no significant difference in improvement was observed between groups.

\section{Abbreviations \\ NP: Neck Pain; SMT: Spinal Manipulative Therapy; HRV: Heart Rate Variability; NDI: Neck Disability Index; NRS-11: 11-point numeric rating scale; HRQoL: Health-related quality of life; LOCF: Last Observation Carried Forward; MCID: Minimal Important Clinical Difference.}

\section{Supplementary Information}

The online version contains supplementary material available at https://doi. org/10.1186/s12891-021-04772-x.

Additional file 1. Graph NRS11. Daily development of mean pain intensity (NRS-11) with Confidence Intervals for both groups from baseline to the end of the intervention period ( 2 weeks). The graph is presented using a quadratic model.

Additional file 2.. Graph McGill. McGill scores with Confidence Intervals for both groups measured at baseline, 1 week, and 2 weeks. The graph is presented using a quadratic model.

Additional file 3. Graph NDI. Neck Disability Index scores with Confidence Intervals for both groups measured at baseline, 1 week and 2 weeks. The graph is presented using a quadratic model.

Additional file 4. Graph EQ5D. EQ5D scores with Confidence Intervals for both groups measured at baseline, 1 week, and 2 weeks. The graph is presented using a quadratic model.

Additional file 5. Stretch exercises to perform daily for 14 days.

\section{Acknowledgments}

A special thanks to www.kiropraktorcentrum.com for the permission to use their photos in the exercise diary.

\section{Authors' contributions}

AGB designed the study, performed the main statistical analysis, prepared tables and wrote the main manuscript text. IA designed the study and assisted in the writing of the manuscript. AE assisted in designing the study and writing of the manuscript. SO assisted in designing the study and reviewed the manuscript. AW assisted in performing the statistical analysis, prepared figures, and reviewed the manuscript's statistics section. The author(s) read and approved the final manuscript.

\section{Authors' information}

This article is the first of a series of three articles as part of AGBs' doctoral degree. The overall aim is to investigate the relationship between changes in pain and changes in HRV.

\section{Funding}

Financial support to this article was provided by IKON - (Institute for Chiropractic and Neuromusculoskeletal Research). The funding body had no role in the design of the study, analysis or interpretation of data, or writing of the manuscript. Open access funding provided by Karolinska Institute.

\section{Availability of data and materials}

The data that support the findings of this study are available from Karolinska Institutet, but restrictions apply to the availability of these data, which were used under license for the current study, and so are not publicly available. Data are, however, available from the authors upon reasonable request and with permission of Karolinska Institutet. 


\section{Declarations}

Ethics approval and consent to participate

Signed informed consent was obtained from all participants. This study was approved by the Regional Ethical Swedish Ethical Review Authority [59] (reference approval no. 2018/2137-31).

\section{Consent for publication}

Consent for the use of the photos of home stretching exercises was obtained.

\section{Competing interests}

The authors declare that they have no competing interests.

\section{Author details}

'Department of Environmental Medicine, Division of Intervention and Implementation Research for Worker Health, Karolinska Institutet, Nobels väg 13, S-, 17177 Stockholm, Sweden. 'Division of Biostatistics, Karolinska Institutet, Nobels väg 13, S-, 17177 Stockholm, Sweden. ${ }^{3}$ Spine Centre Southern Denmark, University Hospital of Southern Denmark, Østre Hougvej 55, 5500 Middelfart, Denmark.

Received: 16 April 2021 Accepted: 4 October 2021

Published online: 27 October 2021

\section{References}

1. Vos T, Abajobir AA, Abate KH, Abbafati C, Abbas KM, Abd-Allah F, et al. Global, regional, and national incidence, prevalence, and years lived with disability for 328 diseases and injuries for 195 countries, 1990\&\#x2013;2016: a systematic analysis for the global burden of disease study 2016. Lancet. 2017;390(10100):1211-59.

2. Hoy DG, Smith E, Cross M, Sanchez-Riera L, Blyth FM, Buchbinder R, et al. Reflecting on the global burden of musculoskeletal conditions: lessons learnt from the global burden of disease 2010 study and the next steps forward. Ann Rheum Dis. 2015;74(1):4-7.

3. March L, Smith EUR, Hoy DG, Cross MJ, Sanchez-Riera L, Blyth F, et al. Burden of disability due to musculoskeletal (MSK) disorders. Best Pract Res Clin Rheumatol. 2014;28(3):353-66.

4. Treede R-D, RiefW, Barke A, Aziz Q, Bennett MI, Benoliel R, et al. A classification of chronic pain for ICD-11. Pain. 2015;156(6):1003-7.

5. Dahlhamer J, Lucas J, Zelaya C, Nahin R, Mackey S, DeBar L, et al. Prevalence of chronic pain and high-impact chronic pain among adults United States, 2016. MMWR Morb Mortal Wkly Rep. 2018;67(36):1001-6.

6. Hoy D, March L, Woolf A, Blyth F, Brooks P, Smith E, et al. The global burden of neck pain: estimates from the global burden of disease 2010 study. Ann Rheum Dis. 2014;73(7):1309-15.

7. Côté P, Cassidy JD, Carroll LJ, Kristman V. The annual incidence and course of neck pain in the general population: a population-based cohort study. Pain. 2004;112(3):267-73.

8. Breivik H, Collett B, Ventafridda V, Cohen R, Gallacher D. Survey of chronic pain in Europe: prevalence, impact on daily life, and treatment. Eur J Pain. 2006;10(4):287-333.

9. Bergström G, Bodin L, Bertilsson H, Jensen IB. Risk factors for new episodes of sick leave due to neck or back pain in a working population. A prospective study with an 18-month and a three-year follow-up. Occup Environ Med. 2007;64(4):279-87.

10. Hurwitz EL, Goldstein MS, Morgenstern H, Chiang LM. The impact of psychosocial factors on neck pain and disability outcomes among primary care patients: results from the UCLA neck pain study. Disabil Rehabil. 2006;28(21):1319-29.

11. Rezai M, Côté P, Cassidy JD, Carroll L. The association between prevalent neck pain and health-related quality of life: a cross-sectional analysis. Eur Spine J. 2008;18(3):371.

12. Evans $G$. Identifying and treating the causes of neck pain. Med Clin. 2014;98(3):645-61.

13. Guez M, Hildingsson C, Nilsson M, Toolanen G. The prevalence of neck pain: a population-based study from northern Sweden. Acta Orthop Scand. 2002;73(4):455-9.
14. Misailidou V, Malliou P, Beneka A, Karagiannidis A, Godolias G. Assessment of patients with neck pain: a review of definitions, selection criteria, and measurement tools. J Chiropractic Med. 2010;9(2):49-59.

15. Treede RD, RiefW, Barke A, Aziz Q, Bennett MI, Benoliel R, et al. Chronic pain as a symptom or a disease: the IASP classification of chronic pain for the international classification of diseases (ICD-11). Pain. 2019;160(1):19-27.

16. Geneen LJ, Moore RA, Clarke C, Martin D, Colvin LA, Smith BH. Physical activity and exercise for chronic pain in adults: an overview of Cochrane reviews. Cochr Database Syst Rev. 2017;1:Cd011279.

17. Law RYW, Harvey LA, Nicholas MK, Tonkin L, De Sousa M, Finniss DG. Stretch exercises increase tolerance to stretch in patients with chronic musculoskeletal pain: a randomized controlled trial. Phys Ther. 2009;89(10):1016-26.

18. Miller J, Gross A, D'Sylva J, Burnie SJ, Goldsmith CH, Graham N, et al. Manual therapy and exercise for neck pain: a systematic review. Man Ther. 2010;15(4):334-54.

19. Hurwitz EL, Carragee EJ, van der Velde G, Carroll LJ, Nordin M, Guzman J, et al. Treatment of neck pain: noninvasive interventions: results of the bone and joint decade 2000-2010 task force on neck pain and its associated disorders. Spine (Phila Pa 1976). 2008;33(4 Suppl):S123-52.

20. Bussieres AE, Stewart G, Al-Zoubi F, Decina P, Descarreaux M, Hayden J, et al. The treatment of neck pain-associated disorders and whiplashassociated disorders: a clinical practice guideline. J Manip Physiol Ther. 2016;39(8):523-64.e27.

21. Bryans R, Decina P, Descarreaux M, Duranleau M, Marcoux H, Potter $B$, et al. Evidence-based guidelines for the chiropractic treatment of adults with neck pain. J Manipul Physiol Therapeut. 2014;37(1):42-63.

22. Blanpied PR, Gross AR, Elliott JM, Devaney LL, Clewley D, Walton DM, et al. Neck pain: revision 2017. J Orthopaed Sports Phys Ther. 2017:47(7):A1-a83.

23. Parikh P, Santaguida P, Macdermid J, Gross A, Eshtiaghi A. Comparison of CPG's for the diagnosis, prognosis and management of nonspecific neck pain: a systematic review. BMC Musculoskelet Disord. 2019;20(1):81.

24. Kvammen OC, Leboeuf-Yde C. The chiropractic profession in Norway 2011. Chiropractic Manual Ther. 2014;22(1):44.

25. Galaasen Bakken A, Axen I, Eklund A, O'Neill S. The effect of spinal manipulative therapy on heart rate variability and pain in patients with chronic neck pain: a randomized controlled trial. Trials. 2019;20(1):590.

26. Hallman DM, Olsson EMG, von Schéele B, Melin L, Lyskov E. Effects of heart rate variability biofeedback in subjects with stress-related chronic neck pain: a pilot study. Appl Psychophysiol Biofeedback. 2011;36(2):71-80.

27. Furlan AD, Yazdi F, Tsertsvadze A, Gross A, Van Tulder M, Santaguida L, et al. A systematic review and meta-analysis of efficacy, cost-effectiveness, and safety of selected complementary and alternative medicine for neck and low-back pain. Evid-based Complement Altern Med. 2012;2012:953139.

28. IBM Corp. Released 2021. IBM SPSS Statistics for Windows, Version 28.0. Armonk: IBM Corp.

29. Leboeuf-Yde C, Axén I, Jones JJ, Rosenbaum A, Løvgren PW, Halasz $L$, et al. The Nordic back pain subpopulation program: the long-term outcome pattern in patients with low back pain treated by chiropractors in Sweden. J Manip Physiol Ther. 2005;28(7):472-8.

30. Cleland JA, Childs JD, Fritz JM, Whitman JM, Eberhart SL. Development of a clinical prediction rule for guiding treatment of a subgroup of patients with neck pain: use of thoracic spine manipulation, exercise, and patient education. Phys Ther. 2007;87(1):9-23.

31. Ylinen J, Kautiainen $H$, Wiren $K$, Hakkinen A. Stretching exercises vs manual therapy in treatment of chronic neck pain: a randomized, controlled cross-over trial. J Rehabil Med. 2007;39(2):126-32.

32. Roenz D, Broccolo J, Brust S, Billings J, Perrott A, Hagadorn J, et al. The impact of pragmatic vs. prescriptive study designs on the outcomes of low back and neck pain when using mobilization or manipulation techniques: a systematic review and meta-analysis. J Man Manip Ther. 2018;26(3):123-35

33. Coulter ID, Crawford C, Vernon H, Hurwitz EL, Khorsan R, Booth MS, et al. Manipulation and mobilization for treating chronic nonspecific neck pain: a systematic review and meta-analysis for an appropriateness panel. Pain Phys. 2019;22(2):E55-e70. 
34. Maiers M, Bronfort G, Evans R, Hartvigsen J, Svendsen K, Bracha Y, et al. Spinal manipulative therapy and exercise for seniors with chronic neck pain. Spine J. 2014;14(9):1879-89.

35. Williamson A, Hoggart B. Pain: a review of three commonly used pain rating scales. J Clin Nurs. 2005;14(7):798-804.

36. Jensen MP, Karoly P, Braver S. The measurement of clinical pain intensity: a comparison of six methods. Pain. 1986:27(1):117-26.

37. Dworkin RH, Turk DC, Trudeau JJ, Benson C, Biondi DM, Katz NP, et al. Validation of the short-form McGill pain Questionnaire-2 (SF-MPQ-2) in acute low back pain. J Pain. 2015;16(4):357-66.

38. Burckhardt CS, Bjelle A. A Swedish version of the short-form McGill pain questionnaire. Scand J Rheumatol. 1994;23(2):77-81.

39. Dansie EJ, Turk DC. Assessment of patients with chronic pain. Br J Anaesth. 2013;111(1):19-25.

40. Bjork S, Norinder A. The weighting exercise for the Swedish version of the EuroQol. Health Econ. 1999;8(2):117-26.

41. Brooks R. EuroQol: the current state of play. Health Policy (Amsterdam, Netherlands). 1996;37(1):53-72.

42. Ackelman $B H$, Lindgren U. Validity and reliability of a modified version of the neck disability index. J Rehabil Med. 2002;34(6):284-7.

43. Vartiainen $P$, Mäntyselkä P, Heiskanen T, Hagelberg N, Mustola S, Forssell $\mathrm{H}$, et al. Validation of EQ-5D and 15D in the assessment of health-related quality of life in chronic pain. Pain. 2017;158(8):1577-85.

44. Bhadhuri A, Kind P, Salari P, Jungo KT, Boland B, Byrne S, et al. Measurement properties of EQ-5D-3L and EQ-5D-5L in recording self-reported health status in older patients with substantial multimorbidity and polypharmacy. Health Qual Life Outcomes. 2020;18(1):317.

45. SMS-track. Available from: https://www.sms-track.com. Retrieved 15 of October 2021

46. KI Survey. Available from: https://www.artologik.com/en/SurveyAndR eport.aspx. Retrieved 15 of October 2021.

47. Saunders J, Wainwright P. Risk, Helsinki 2000 and the use of placebo in medical research. Clin Med (London, England). 2003;3(5):435-9.

48. Charlton E. Ethical guidelines for pain research in humans. Committee on ethical issues of the International Association for the Study of Pain. Pain. 1995;63(3):277-8

49. Van Buuren S, Brand JPL, Groothuis-Oudshoorn CGM, Rubin DB. Fully conditional specification in multivariate imputation. J Stat Comput Simul. 2006;76(12):1049-64.
50. Farrar JT, Young JP Jr, LaMoreaux L, Werth JL, Poole RM. Clinical importance of changes in chronic pain intensity measured on an 11-point numerical pain rating scale. Pain. 2001;94(2):149-58.

51. Hawker GA, Mian S, Kendzerska T, French M. Measures of adult pain: visua analog scale for pain (VAS pain), numeric rating scale for pain (NRS pain), McGill pain questionnaire (MPQ), short-form McGill pain questionnaire (SF-MPQ), chronic pain grade scale (CPGS), short Form-36 bodily pain scale (SF-36 BPS), and measure of intermittent and constant osteoarthritis pain (ICOAP). Arthritis Care Res. 2011;63(Suppl 11):S240-52.

52. Soer R, Reneman MF, Speijer BL, Coppes MH, Vroomen PC. Clinimetric properties of the EuroQol-5D in patients with chronic low back pain. Spine J. 2012;12(11):1035-9.

53. Young BA, Walker MJ, Strunce JB, Boyles RE, Whitman JM, Childs JD. Responsiveness of the neck disability index in patients with mechanical neck disorders. Spine J. 2009;9(10):802-8.

54. Rubinstein SM, Leboeuf-Yde C, Knol DL, de Koekkoek TE, Pfeifle CE, van Tulder MW. The benefits outweigh the risks for patients undergoing chiropractic care for neck pain: a prospective, multicenter, cohort study. J Manip Physiol Ther. 2007;30(6):408-18.

55. Myhrvold BL, Kongsted A, Irgens P, Robinson HS, Thoresen M, Vollestad NK. Broad external validation and update of a prediction model for persistent neck pain after 12 weeks. Spine (Phila Pa 1976). 2019;44(22):E1298-e310.

56. Carnes D, Mars TS, Mullinger B, Froud R, Underwood M. Adverse events and manual therapy: a systematic review. Man Ther. 2010;15(4):355-63.

57. Friedrich M, Cermak T, Maderbacher P. The effect of brochure use versus therapist teaching on patients performing therapeutic exercise and on changes in impairment status. Phys Ther. 1996;76(10):1082-8.

58. Medina-Mirapeix F, Escolar-Reina P, Gascón-Cánovas JJ, Montilla-Herrador J, Jimeno-Serrano FJ, Collins SM. Predictive factors of adherence to frequency and duration components in home exercise programs for neck and low back pain: an observational study. BMC Musculoskelet Disord. 2009:10(1):1-9.

59. Available from: https://etikprovningsmyndigheten.se/.

\section{Publisher's Note}

Springer Nature remains neutral with regard to jurisdictional claims in published maps and institutional affiliations.
Ready to submit your research? Choose BMC and benefit from:

- fast, convenient online submission

- thorough peer review by experienced researchers in your field

- rapid publication on acceptance

- support for research data, including large and complex data types

- gold Open Access which fosters wider collaboration and increased citations

- maximum visibility for your research: over $100 \mathrm{M}$ website views per year

At BMC, research is always in progress.

Learn more biomedcentral.com/submissions 